\title{
Sistemas de gamificación pregunta-respuesta para la evaluación de competencias en el área de Fisiología.
}

\section{Question-answer gamification systems for the assessment of competences in the area of Physiology.}

\author{
Rafael Pineda Reyes ${ }^{1, \# \text {, Juan Manuel Castellano Rodríguez }}{ }^{2, \#, ~ J u a n ~ R o a ~}$ \\ Rivas $^{3, \#, ~ L e o n o r ~ P i n i l l a ~ J u r a d o ~}{ }^{4}$, Francisco Gaytán Luna ${ }^{5}$, Manuel Tena- \\ Sempere ${ }^{6}$
}

Fecha de recepción: 13/10/2019; Fecha de revisión: 05/11/2019; Fecha de aceptación: 29/11/2019

Cómo citar este artículo:

Pineda, R., Castellano, J.M., Roa, J., Pinilla, L., Gaytán, F. \& Tena-Sempere, M. (2019). Sistemas de gamificación pregunta-respuesta para la evaluación de competencias en el área de Fisiología. Revista de Innovación y Buenas Prácticas Docentes, 8(4), 116-123.

Autor de Correspondencia: fi1tesem@uco.es

\section{Resumen:}

El presente proyecto ha consistido en la implantación de un sistema de gamificación de preguntas y respuestas en clase con el objetivo de mejorar la motivación y el compromiso de los alumnos con el aprendizaje en las asignaturas del área de Fisiología: Fisiología General (Grado de Medicina, 180 alumnos), Fisiología (Grado de Enfermería: 170 alumnos) y Fisiología Especial (Grado de Fisioterapia: 70 alumnos). Como sistema de gamificación se empleó la aplicación web Kahoot!, que permite registrar en tiempo real, mediante el uso de dispositivos remotos individuales (p.ej. teléfonos móviles), la participación de los estudiantes en las diferentes tareas propuestas (p.ej. preguntas tipo test, evaluaciones). Los datos registrados nos permiten concluir que los alumnos han conseguido un grado de motivación significativamente superior al observado en cursos anteriores, en los que este sistema aún no se había implantado. Además, consideramos que dicha motivación viene dada por el papel protagonista que han adquirido los estudiantes tras la implantación de este nuevo sistema. En resumen, estos resultados sugieren que la gamificación es una herramienta muy útil para estimular la motivación de los estudiantes y abre la posibilidad de su implantación en otros contextos educativos, especialmente en aquellos en los que los estudiantes no se sientan motivados.

Palabras clave: Fisiología, gamificación, Kahoot!, teléfonos móviles.

\footnotetext{
${ }_{1}^{1}$ Universidad de Córdoba (España), v92pirer@uco.es; CÓDIGO ORCID: 0000-0001-6769-3575

2 Universidad de Córdoba (España), b82caroj@uco.es; CÓDIGO ORCID: 0000-0003-3981-6683

${ }^{3}$ Universidad de Córdoba (España), roarivas@gmail.com; CÓDIGO ORCID: 0000-0002-8970-421X

4 Universidad de Córdoba (España), bc1pijul@uco.es; CÓDIGO ORCID: 0000-0002-5897-4057

${ }^{5}$ Universidad de Córdoba (España), bc1galuf@uco.es; CÓDIGO ORCID: 0000-0002-3066-126X

${ }^{6}$ Universidad de Córdoba (España), fi1tesem@uco.com; CÓDIGO ORCID: 0000-0002-4741-5567

\# Estos autores contribuyeron de igual manera a la realización del trabajo y deben ser considerados autores principales.
} 
Abstract:

The present project consisted of the implementation of a question-answer gamification system in class in order to improve the motivation and engagement of the students in learning subjects of Physiology: General Physiology (Degree in Medicine, 180 students), Physiology (Degree in Nursing, 170 students), Special Physiology (Degree in Physiotherapy, 70 students). We used a web application, named Kahoot!, as a gamification system. This application allows, through the use of remote control devices (e.g., mobile phones), real time recording of student's involvement in the proposed tasks (e.g. tests, assessments). Based on our recorded data, we can conclude that students have attained a higher degree of motivation than in previous years, where this system had not been implemented. Furthermore, we believe that such improvement is due to the leading role acquired by the students in this new system. In sum, these results suggest that gamification is a useful and stimulating tool for student's motivation and open the possibility for its application in other educational contexts, especially those where the students are not motivated.

Key Words: Physiology, Gamification, Kahoot!, mobile phone 


\section{INTRODUCCIÓN}

El sistema educativo universitario tradicional es percibido por algunos estudiantes como un sistema ineficiente y aburrido (McIntyre \& Munson, 2008); un hecho que fomenta la búsqueda de nuevos enfoques educativos innovadores que favorezcan la motivación y el compromiso de los alumnos con el aprendizaje (Bruff, 2009; Duncan, 2005). En este contexto, el uso de juegos educativos como herramientas de aprendizaje, en entornos no lúdicos como la Universidad, podría ofrecer un enfoque muy prometedor que reforzaría no sólo los conocimientos de los alumnos, sino también sus habilidades para solventar problemas, colaborar y comunicarse en clase (Bruff, 2009; Duncan, 2005). Además, los juegos tienen un destacable componente motivacional, ya que emplean una serie de mecanismos que animan a la participación por el mero hecho de disfrutar del juego y tener la oportunidad de ganar, incluso aunque no se establezca ningún tipo de recompensa, más allá de la satisfacción personal asociada al reconocimiento de las cualidades cognitivas del alumno (Bruff 2009; Duncan 2005). Sin embargo, los juegos educativos convencionales no sólo son difíciles de desarrollar, sino que también suponen una gran inversión de tiempo, dinero y esfuerzo. En este contexto, ha surgido un nuevo procedimiento de juego conocido como ludificación o "gamificación" (término anglosajón) (Kapp, 2012). Este procedimiento fue definido por Deterding y colaboradores (2011) como "el uso de elementos de diseño de juegos en contextos no recreativos". La gamificación, a diferencia del juego educativo convencional, no requiere de la creación, desarrollo e implementación de todos los componentes del juego, sino de unos elementos específicos, resultando no sólo más rentable que el juego educativo convencional (en términos de tiempo, esfuerzo y costes), sino también igualmente efectivo en lo que se refiere a motivación y compromiso de los alumnos con el aprendizaje (Kapp, 2012).

La gamificación se ha expandido recientemente en el mundo de los negocios, el marketing, la gestión corporativa y diferentes iniciativas ecológicas. Esto se debe, en gran medida, a su potencial para configurar el comportamiento de los usuarios en la dirección deseada (Kapp, 2012). Este tipo de estrategias ya se aplica con éxito en diferentes sitios web de educación online, tales como codeacademy.com y khanacademy.org, los cuales emplean la gamificación para estimular a sus usuarios. Así, cuanto mayor es el número de lecciones y cursos completados, más credenciales consiguen los usuarios y más se refuerza su motivación.

En base a lo expuesto anteriormente, nos planteamos implantar un sistema de gamificación pregunta-respuesta en las clases de Fisiología, Fisiología General y Fisiología Especial correspondientes a los Grados Medicina, Enfermería y Fisioterapia, con el objetivo de: (i) identificar el conocimiento previo de los estudiantes en contenidos relacionados con el área de Fisiología; (ii) aumentar el compromiso de los estudiantes y su participación en clase; (iii) facilitar el aprendizaje y el pensamiento critico; (iv) evaluar el aprendizaje de los estudiantes durante el desarrollo de las clases; ( $v$ ) ayudar a los estudiantes y a los propios docentes a identificar posibles lagunas en el aprendizaje de los contenidos impartidos en el área de Fisiología; y (vi) preparar a los estudiantes en el ejercicio de la valoración de casos clínicos. 
Pensamos que la implantación de este sistema podría suponer una mejora significativa en aspectos muy relevantes del aprendizaje de los estudiantes, que implican no sólo su asimilación de contenidos y motivación, sino también su preparación como profesionales clínicos.

\section{DESARROLLO DE LA EXPERIENCIA DE INNOVACIÓN}

La implantación del sistema de gamificación se llevó a cabo en las clases de Fisiología General, Fisiología y Fisiología Especial correspondientes a tres grados diferentes; Medicina (Primer curso, con unos 180 alumnos matriculados), Enfermería (Primer curso, con unos 170 alumnos matriculados) y Fisioterapia (Primer curso, con unos 70 alumnos matriculados). Este sistema de gamificación pregunta-respuesta se desarrolló a través de la aplicación web Kahoot! (http://www.kahoot.com); una aplicación que permite utilizar los dispositivos móviles en el aula como soporte para la clase. Esta aplicación registra en tiempo real la participación de los estudiantes (asistencia) en las diferentes tareas propuestas (p.ej. tests, evaluaciones, casos clínicos, etc...), permitiendo de este modo la gestión inmediata de la información registrada y la monitorización del aprendizaje de los alumnos por parte del profesorado. Además, Kahoot! contempla un acceso diferenciado para alumnos y profesores, preservando la confidencialidad de la información gestionada por parte del profesorado.

En relación a las actividades a desarrollar, conviene destacar que Kahoot! permitió que el profesorado estableciese (i) cuestionarios sin límites de tiempo de respuesta, (ii) cuestionarios de tiempo limitado o (iii) cuestionarios con ranking de resultados. Además, el profesorado pudo seguir los resultados en directo y/o revisarlos posteriormente en los informes de hoja de datos Excel que se almacenan en la aplicación. Los tipos de preguntas que se aplicaron en clase fueron las siguientes:

Preguntas de repaso.

- Preguntas conceptuales de comprensión.

- Preguntas de aplicación de los contenidos impartidos.

- Preguntas relacionadas con el desarrollo del pensamiento crítico.

- Preguntas sobre la perspectiva del estudiante.

- Preguntas sobre el nivel de confianza.

- Preguntas de monitorización.

- Preguntas en pareja.

- Preguntas repetidas.

Los test con las preguntas se realizaron antes, durante y al finalizar las distintas unidades didácticas impartidas en las diferentes asignaturas, de forma que permitiera el seguimiento continuo de la adquisición de conocimientos por parte del alumnado. En general, estas preguntas permitieron evaluar la asimilación de los principales conceptos expuestos en clase, su aplicación en casos clínicos y la capacidad del 
alumno para trabajar en equipo y desarrollar su pensamiento crítico de cara a la resolución de las cuestiones planteadas.

La consecución del proyecto propició el desarrollo de una serie de competencias básicas (CB), propias de Universidad (CU) y transversales (CT). Entre ellas destacan:

CB1: Expresarse correctamente de manera oral y escrita en castellano.

CB4: Aplicar los conocimientos adquiridos y su capacidad de resolución de problemas en entornos nuevos o poco conocidos dentro de contextos más amplios (o multidisciplinares) relacionados con su área de estudio.

CB6: Transmitir información, ideas, problemas y soluciones y que sepan comunicar sus conclusiones y los conocimientos y razones que las sustentan, a públicos especializados y no especializados de un modo claro y sin ambigüedades.

CU2: Conocer y perfeccionar el nivel de usuario en el ámbito de las TICs.

CT1: Análisis objetivo y la capacidad de síntesis.

CT11: Capacidad crítica y autocrítica.

CT12: Desarrollo de la capacidad de trabajo en equipo.

Además de las competencias indicadas como ejemplos, también se desarrollaron competencias específicas relacionados con la materia de Fisiología (competencias específicas de cada grado).

\section{RESULTADOS}

La aplicación del sistema de gamificación pregunta-respuesta propuesto en este proyecto ha contribuido a mejorar de forma significativa el aprendizaje de los estudiantes, estimulando especialmente su atención y motivación durante la impartición de las clases y/o seminarios. Esto es debido a que este sistema favorece la participación activa del alumno en clase, haciéndole disfrutar del juego y de la recompensa intelectual asociada a la contestación adecuada de las preguntas/actividades propuestas. Por otra parte, y como se puede observar, a modo de ejemplo representativo en las tablas que se describen a continuación (Figura 1), los resultados obtenidos pusieron de manifiesto la mejora en los porcentajes de acierto de los estudiantes tras la asimilación de los contenidos y el desarrollo de distintos test de forma consecutiva. Así, en el ejemplo de la Figura 1 se puede observar como el porcentaje de respuestas correctas paso del $62,85 \%$ al $89,12 \%$, una mejora del $26 \%$, en el caso del bloque sobre el Sistema Endocrino, tras la realización del segundo test y en los temas sobre las Glándulas Adrenales el porcentaje de respuestas correctas paso del $77,65 \%$ al $87,21 \%$; una mejora de cerca del $10 \%$. Estos porcentajes de mejora indican una clara progresión del alumnado en la adquisición de nuevos conocimientos. Aunque los ejemplos que se muestran en la Figura 1 son los correspondientes a los contenidos relacionados con el Sistema Endocrino y las Glándulas Adrenales, estos resultados se repitieron de forma consistente en el resto de asignaturas y grados. Esto nos permitió, por un lado, evaluar los conocimientos 
previos del alumnado, antes del comienzo de los distintos bloques de la asignatura, para así ajustar los contenidos a las necesidades generales de la clase. Y, por otro lado, también nos permitió analizar la asimilación de contenidos por parte de los alumnos, tras la impartición de éstos en las distintas secciones de las asignaturas.
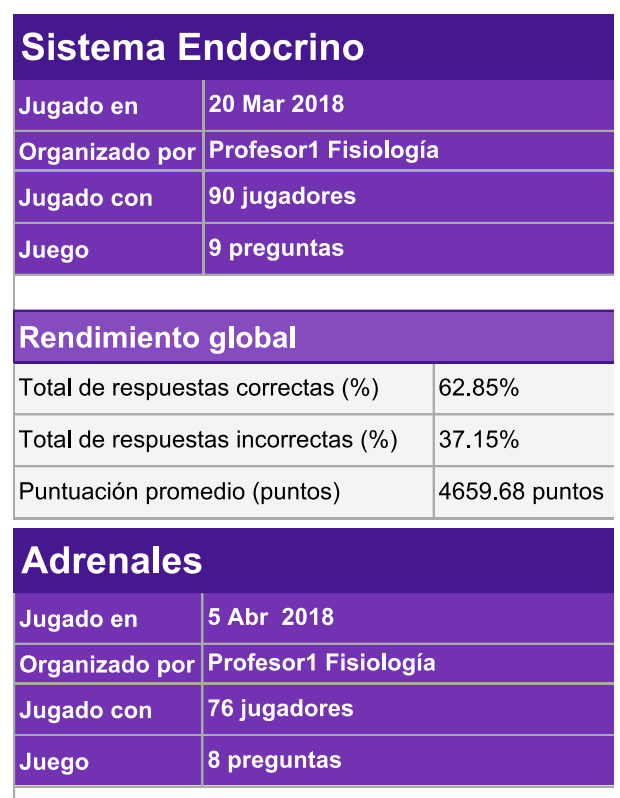

\section{Rendimiento global}

\begin{tabular}{|l|l|}
\hline Total de respuestas correctas (\%) & $77.65 \%$
\end{tabular}

\begin{tabular}{l|l} 
Total de respuestas incorrectas (\%) & $22.35 \%$
\end{tabular}

\begin{tabular}{l|l} 
Puntuación promedio (puntos) $\quad 6156.34$ puntos
\end{tabular}

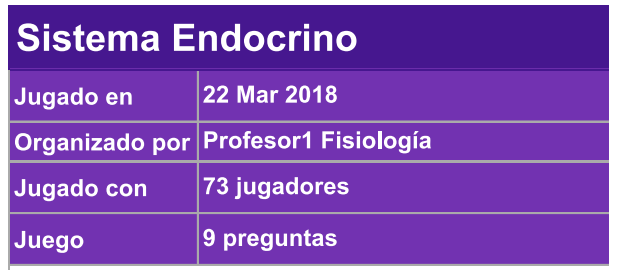

\begin{tabular}{|l|l|}
\hline Rendimiento global \\
\hline Total de respuestas correctas (\%) & $89.12 \%$ \\
\hline Total de respuestas incorrectas (\%) & $10.88 \%$ \\
\hline Puntuación promedio (puntos) & 8657.67 points \\
\hline
\end{tabular}

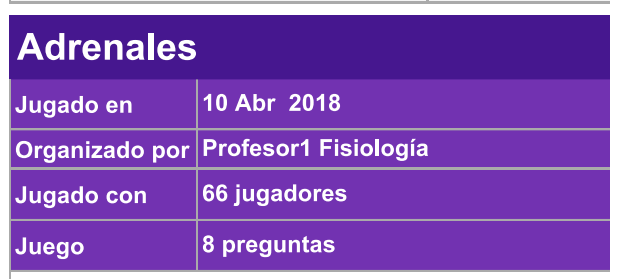

Rendimiento global

Total de respuestas correctas (\%) $\quad 87.21 \%$

\begin{tabular}{|l|l}
\hline Total de respuestas incorrectas (\%) & $12.79 \%$
\end{tabular}

\begin{tabular}{|l|l|}
\hline Puntuación promedio (puntos) & 7573.39 puntos
\end{tabular}

Figura 1: Evolución del porcentaje de aciertos y fallos al inicio (paneles de la izquierda) y finalización (paneles de la derecha) de los contenidos impartidos sobre el sistema endocrino (paneles superiores) y las glándulas adrenales (paneles inferiores).

Además, también mejoró el pensamiento crítico y la asimilación de contenidos, gracias a la selección concienzuda de las preguntas y al análisis exhaustivo de las respuestas, que animó a que los alumnos reflexionasen y asimilaran el contenido de la materia durante la clase. Del mismo modo, también mejoró el debate y la colaboración en clase, mediante el desarrollo de ejercicios en grupo que requirieron que los estudiantes discutiesen y llegasen a un consenso. Otro hecho importante en relación a esto es que se favoreció la participación de todos los estudiantes de la clase, ya que estas actividades de gamificación, que únicamente requieren de la disponibilidad de dispositivos móviles, ofreció a los estudiantes la oportunidad de responder en silencio y de forma privada, permitiendo de este modo que aquellos estudiantes que no suelen participar en clase también pudieran expresar sus pensamientos y opiniones. Adicionalmente, el sistema de gamificación favoreció que los alumnos pensaran y registraran sus respuestas en un tiempo delimitado, estimulando su agilidad mental. Por otra parte, estos sistemas también favorecieron que los estudiantes se animaran a responder a cuestiones éticas, legales y morales, ya que al hacerlo de forma anónima se sentían más libres para expresarse. 
En relación al profesorado, conviene destacar que la gamificación permitió la evaluación inmediata del aprendizaje de los estudiantes durante la clase, ya que los profesores pudieron comprobar si los estudiantes estaban asimilando los contenidos impartidos durante el desarrollo de las clases, sin tener que esperar a los resultados de los exámenes para confirmar lo que habían aprendido. De este modo se aclararon conceptos que no habían sido entendidos durante la clase; un hecho que pensamos ha podido contribuir a mejorar el porcentaje de alumnos que aprobaron la materia en los exámenes finales. En este sentido, pensamos que la posibilidad de conocer en tiempo real los resultados de las actividades de gamificación le ha permitido al profesorado adaptarse de forma inmediata a las necesidades de aprendizaje de los estudiantes. De hecho, cuando el histograma de respuestas revelaba que un número significativo de estudiantes había seleccionado la respuesta incorrecta a la pregunta planteada, el profesor pudo identificar dicho problema y volver a explicar o aclarar los puntos que no habían quedado claros. Del mismo modo, si el histograma mostraba que la mayoría de los estudiantes elegían las respuestas correctas a las preguntas formuladas, el profesorado consideró que los conceptos básicos habían sido asimilados adecuadamente y se podía continuar la materia con normalidad.

\section{CONCLUSIONES}

Los resultados obtenidos en este proyecto indican un alto grado de participación del alumnado en las actividades de gamificación y una mejora significativa en los porcentajes de acierto tras la asimilación de los contenidos. Estos datos sugieren que las actividades de gamificación favorecen una actitud más activa por parte de los alumnos y un reforzamiento de la atención y la motivación en clase. Además, los debates que se crearon en torno a las preguntas planteadas mejoraron, no sólo el pensamiento crítico a la hora de resolver las cuestiones, sino también la comunicación entre alumnos y el diálogo entre los alumnos y el profesor, generando un ambiente ideal para la confianza y el aprendizaje. De hecho, fue muy interesante asistir y participar en los diálogos planteados por los propios estudiantes, ya que solían ofrecer alguna alternativa interesante en relación a las cuestiones planteadas, que defendían posteriormente de forma argumentada.

En base a estos resultados, podemos concluir que la gamificación es una experiencia muy útil, que podría aplicarse no sólo en el contexto de la enseñanza de Fisiología en el ámbito universitario, sino también en otras áreas de conocimiento y en diferentes niveles de enseñanza.

\section{REFERENCIAS}

Bruff, D. (2009). Teaching with classroom response systems: Creating active learning environments. San Francisco: Jossey-Bass.

Deterding, S., Khaled, R., Nacke, L.E. \& Dixon, D. (2011). Gamification: Toward a Definition. In CHI 2011 Gamification Workshop Proceedings, Vancouver, BC, Canada.

Duncan, D. (2005). Clickers in the classroom: How to enhance science teaching using classroom response systems. San Francisco: Pearson Education.

Kapp, K. M. (2012). The gamification of learning and instruction: game-based methods and strategies for training and education. John Wiley \& Sons. 
Mclntyre, S. H. \& Munson, J.M. (2008). Exploring Cramming: Student Behaviors, Beliefs, and Learning Retention in the Principles of Marketing Course. Journal of Marketing Education, 30 (3), 226-243. 